(C2008 IEEE. Personal use of this material is permitted. However, permission to reprint/republish this material for advertising or promotional purposes or for creating new collective works for resale or redistribution to servers or lists, or to reuse any copyrighted component of this work in other works must be obtained from the IEEE 


\section{Ambient Intelligence: A New Multidisciplinary Paradigm}

\begin{abstract}
Ambient intelligence (AmI) is a new multidisciplinary paradigm rooted in the ideas of Norman ${ }^{1}$ and Ubiquitous Computing. AmI fosters novel anthropomorphic human-machine models of interaction. In AmI, technologies are deployed to make computers disappear in the background, while the human user moves into the foreground in complete control of the augmented environment. AmI is a user-centric paradigm, it supports a variety of artificial intelligence methods and works pervasively, nonintrusively, and transparently to aid the user. AmI supports and promotes interdisciplinary research encompassing the technological, scientific and artistic fields creating a virtual support for embedded and distributed intelligence.
\end{abstract}

Index Terms-Ambient intelligence (AmI), artificial intelligence, computer vision, distributed and embedded intelligence.

\section{INTRODUCTION}

I $\mathrm{N}$ the last decade, we have witnessed a more practical and user-centered implementation of computer science research. Virtual and augmented reality are two examples [16] (originated with [20]). Recent trends will affect our lives profoundly by improving the way we live, our social habits, and how we conduct business. Ambient intelligence (AmI) is a new paradigm that supports the design of the next generation of intelligent systems and introduces novel means of communication between human, machine, and the surrounding environment (man-made objects [32]).

AmI [38] provides basic criteria to develop intelligent environments where electronic devices can be so tiny, virtually invisible to us, to disappear into the fabric of our lives [40]. The idea of an environment with pervasive (but not intrusive) computing power was first conceived by Weiser, as he set the grounds for Ubiquitous Computing in the early nineties [44], [45]. In a near future, we could imagine AmI technology assisting the user by autonomously interpreting their intentions. Current technology can already be used to recognize the presence of a person in a private or public space and trigger an automatic response or reaction, for instance, by regulating the temperature and ventilation levels, or transferring phone calls or relaying messages, sorted by urgency or importance precompiled by the user or learnt by observing the user. AmI prototype systems have been implemented and have been tested in controlled conditions [36].

An AmI system can be implemented customized to the needs of the user. The basic idea is of a distributed layered architecture enabling ubiquitous communication and an advanced human-machine communication protocol. A wide range of application domains can benefit from AmI research, including

Digital Object Identifier 10.1109/TSMCA.2004.838456

${ }^{1}$ Author of the Invisible Computer [32].
Health, Managed Public Environments and Practice Skills Training. The AmI paradigm sets the principles to design a pervasive and transparent infrastructure capable of observing people without prying into their lives, adapting to the needs of the user.

In 2001, the European Commission launched the AmI challenge, reported in the document entitled Scenarios for Ambient Intelligence in 2010 [21] written by the IST Programme Advisory Group (ISTAG) for the Fifth European Framework Programme (1998-2002). An updated version of the continuously evolving vision of the report appeared in 2003 under the title Ambient Intelligence: from vision to reality [22], putting forward ideas for the currently open Sixth Framework Programme (2002-2006). Although AmI was coined in Europe, research aimed at the development of Smart Environments has inspired a number of research projects worldwide. To mention a few, there are: the Oxygen Project [12] and the GIT Aware Home [25] in the US, [7] (see also the work of [15]) and the SONY Interaction Laboratory [26] in Japan.

AmI puts forward the criteria for the design of intelligent environments; the implementation process of an advanced AmI system is a very complex technological endeavor. Modern Artificial Intelligence has been used and will be used as it offers distributed solutions to complex problems, stressing the importance of modularity (multiagent systems as teams of intelligent units sensing and interacting to solve independently, in cooperation or competitively aspects of the problem at hand ${ }^{2}$ ). A system based on AmI criteria requires a bundle of technologies to implement modularity, low-power devices, distributed and high band-width heterogeneous networks of sensors and actuators [18].

AmI can benefit from a panoply of research areas, however future implementations of AmI technology will have to rely on machine learning research. Creating complex models for an intelligent system is unrealistic and the necessary mapping of sensory information onto an intelligent and preemptive set of behaviors is by far too complex to be precompiled and hardwired into the system.

AmI sees the human user as the main actor, always in control, playing multiple roles in society. This perhaps is the real new facet of research related to AmI: it introduces a new dimension, creating synergies between the user and the environment, permeating our society, helping the life of disabled people. AmI can potentially enhance the training of professional skills, broadening the learning and training opportunities for students, and making simpler and more pleasant the life of the average citizen at home and in public spaces.

\footnotetext{
${ }^{2}$ Outside the scope of this discussion/introduction would be an in-depth analysis of the term intelligence; the interested reader might consult [37].
} 


\section{PRESEnt Stance}

A large variety of disciplines can be grouped under the umbrella of AmI: distributed intelligence, data and information communication, software design, computer vision, speech recognition, robotics, information fusion, hardware design, computer wearables, social sciences, ethics, and law. AmI is a new paradigm that and a new powerful design theory that can be employed to develop intelligent environments, with eyes and ears. AmI can intuit the user and aid them in their daily chores, in their working space and endeavors. AmI can also monitor the user and can create a safety net around them, making their surroundings more secure and pleasant to live and inhabit.

AmI projects the human user into the foreground and this has profound legal and ethical implications. One can imagine developing an AmI-based system for the training of hospital staff; how intrusive this system can be? What authorities ought to be involved to make the approach/system acceptable? Will we ever be able to convince hospital staff to agree to its installation? What about the patients? Will they have to sign a contract before being accepted in the hospital? AmI is user-centric. System based on the AmI paradigm will have to be human-centric as well, ethical and legal aspects will have to be integrated.

AmI research is already supported by a number of initiatives, including past [2] and forthcoming workshops [4], [5], symposia [1], [3], and book collections [8], [36], [42]. A number of research areas will have to converge to make AmI systems possible. Aart [6] defines various dimensions of the AmI paradigm; hereafter, we discuss what we believe are the hard problems that research will have to focus on in order to meet AmI goals and develop realistic AmI-based systems.

\section{A. Distributed Intelligence}

Distributed intelligence encompasses those techniques that employ modular units of intelligence, known as agents [23], [39], [46], to create a pervasive and distributed layer of intelligence [17]. Intelligent units or agents can be thought of controlling all aspects of an AmI system, from the sensing, to the understanding of information, to the elaboration and execution of behavior in the augmented environment, more or less transparently, with the aim of aiding the user. It would be pointless to describe all the efforts of the multiagent community, worth a note is the research at MIT (the oxygen project) [12], but also and [19], [27], and [30], who implemented distributed solutions.

\section{B. Hardware Design}

For some researchers, AmI is seen as the development of new hardware technologies to enhance people's lives. Although somehow a little limiting, this is partially true. No AmI-based system would work if new hardware solutions were not devised and deployed to equip the smart space with new pervasive hardware, or wearable intelligent solutions [34].

Along this line of work, a number of devices have been proposed. To mention a few: fully integrated meeting rooms [31], with white board and meeting table wired and communicating for fast delivery and transportation of information; wearables to interact with the environment [13]; and intelligent hardware implemented for Edutainment [28], low-power intelligent appliances [43] and objects [41], and augmented reality solutions [16].

\section{Information Understanding}

In order to make use of hardware and to distribute intelligence, information must be extracted from a heterogeneous network of sensors implemented for AmI. The idea is to define what intelligence means in terms of real-time data mining, creating on-the-fly updatable models of the scene, and then generate patterns of intelligence able to migrate over the hardware layer built for the pervasive network [27].

What we might call machine understanding encompasses research in the field of computer vision, dedicated to interpret image sequences [33], [48], speech recognition, dedicated to interpret speech [9], [24], combinations thereof [47] and all those techniques applied by the community working on information fusion [14], [25] and developing intelligent and anthropomorphic user interfaces [35]. The idea is to create an advanced AmI system based on sound research, rooted in various disciplines, dedicated to sensor modeling and analysis, to deploy general models of understanding of combined information for a better understanding of the evolution of a dynamic scene.

\section{Communication Modeling}

Distributing intelligence is possible only if an intelligent layer can be built on top of a robust seamless communication infrastructure (see, for instance, [11]). Once more, data communication is not a new bag of tricks, what is important is to be able to craft techniques better suited for AmI, dedicated to distribute intelligence and create the basis of a new layer of communication. Along this line of work, research exists to create the basis for a transport layer dedicated to intelligence, to create dedicated ontologies [10], [29], and to devise ways of exchanging information and knowledge at the right time. For the latter problem, agent technology might be a solution, even though it will have to be optimized to deal with real-time operation.

\section{SPECIAL ISSUE ON AMI}

Accepted papers cover both theoretical and practical aspects of AmI, describing all development phases of an AmI system. Given the multidisciplinary nature of the proposed topic, we have integrated submissions from a number of research fields for publication: from architectures and user requirements to quality assessment, from sensors and communication requirements to computer vision and artificial intelligence. The focus of this special issue is on the detection of the users within AmI spaces, in particular through video cameras and applying computer vision techniques. This is a fundamental step to create a first level of user-awareness in the environments. The main topics include

- architectures for AmI;

- human factors research for user-requirements design and quality assessment; 
- sensors and communication required for the infrastructure of an AmI system;

- computer vision research, linked with one or more camera sensors, including data fusion problems;

- artificial Intelligence solutions, including scene understanding and creation of simulation;

- AmI solutions for different application domains including health, practical skills training, public spaces management, etc.;

- performance evaluation: can measures be defined to evaluate and compare AmI systems.

The contributions to this special issue are as follows.

"Active Affective State Detection and User Assistance with Dynamic Bayesian Networks," written by X. Li and Q. Ji, presents an intelligent user-assistance system for ubiquitous computing applications which are characterized by ambiguous, uncertain, and multimodal sensory observations; user's changing state; and various constraints on available resources and costs in making decisions. In particular, the authors introduce a new probabilistic framework based on the dynamic Bayesian networks (DBNs) to dynamically model and recognize affective states of the user and to provide the appropriate assistance in order to keep user in a productive state. The main novelty presented in the paper consists of the use of an active sensing mechanism into the DBN framework to integrate different information in order to infer the user's affective state and to provide correct assistance in a timely and efficient manner. The proposed system has been tested on both synthetic and real data. Experimental results demonstrate the feasibility of the proposed framework as well as the effectiveness of the proposed active sensing strategy.

"Control and Learning of Ambience by an Intelligent Building," written by U. Rutishauser, J. Joller, and R. Douglas, focuses on intelligent buildings. Recent studies on working environments emphasize the importance of dynamic reconfiguration of space and function to meet the needs, comfort, and preferences of its inhabitants. Intelligent buildings must have the autonomous capability of satisfying the needs of its inhabitants without human intervention. In this paper, a multiagent control system for a commercial intelligent building equipped with sensors and effectors is presented. Sensors and effectors are linked by means of fuzzy rules and the agents communicate with one another by asynchronous messaging. The main novelty of the paper is an unsupervised online real-time learning algorithm that constructs a fuzzy rule derived from very sparse data in a nonstationary environment. Experimental results are presented to demonstrate that the proposed learning algorithm significantly improve the performance of the building. Specific methods for performance evaluation are also presented.

"Video Security for Ambient Intelligence," written by L. Snidaro, C. Micheloni, and C. Chiavedale, addresses the important issue of security in intelligent spaces. In particular, the work is focused on two features for the security of people in intelligent buildings: people tracking and counting through multiple video sensors. The former can be employed to prevent entry to dangerous or nonauthorized areas, the latter can be used to estimate the number of accesses and as input to regulate other subsystems of the building (i.e., heating, ventilation, and air conditioning) or for statistical purposes. The paper discusses the issues related to the effective implementation of such an application in real conditions, proposing computer vision algorithms, and solutions supported by experimental results in a real test bed. In particular, the performance of the people counting subsystem is discussed in detail reporting results obtained in different conditions.

In "Prismatica: Towards Ambient Intelligence in Public Transport Environments," an automatic visual-based surveillance system for improving safety and security in public transport networks and other public places, is presented by S. Velastin, B. Boghossian, B. Lo, J. Sun, and M. A. VicencioSilva. In particular, the paper introduces a new system architecture that takes into account the distributed nature of the detection process involving different people, management procedures and technology and the need to allow for a different type of devices and actuators. The main innovative aspects of the paper are focused on new algorithms able to integrate object motion in the background/foreground-detection procedure, to generate automatically self-calibration procedures for fixed cameras which are robust to occlusions, camera shake and illumination changes. The system components have been implemented, integrated and tested in a real metropolitan railway environment and they are considered to be a first step toward providing AmI in such complex scenarios.

In "What Planner for Ambient Intelligence Applications?" F. Amigoni, N. Gatti, C. Pinciroli, and M. Roveri discuss the development of an AmI application that effectively adapts the presence of planning mechanisms for goal-oriented behavior to the needs of the users. The authors present a planning system for AmI applications, based on a distributed hierarchical task network approach, the ablility to produce courses of actions to address given goals. The plans produced are flexibly tailored to exploit in the best way the capabilities of the devices currently available in the environment. The main original contributions of the paper are the characterization of the planning problem within AmI, the proposal of a planner for AmI applications that combines centralized and distributed features and the ability of the proposed planner to adapt the planning process and its results to the capabilities of the devices currently connected to the system. Experimental results are presented on a real application scenario in which an AmI system monitors and answers the needs of a diabetic patient.

"An Interactive Space That Learns to Influence Human Behavior," presented by K. Eng, R. J. Douglas, and P. Verschure, tries to answer a key question in the design of intelligent environments: how a space can influence the actions of its users, and how such behavior can be learned. The authors describe the re- 
sults of experiments conducted in an interactive entertainment exhibit deployed at the 2002 Swiss National Exhibition (Expo 02). The main innovative part of the paper is a new learning model called distributed adaptive control that is based on the animal learning paradigms of classical and operant conditioning and that has been developed using mobile robots in foraging tasks. The proposed model was applied to the learning of effective cues for guiding visitors in a given direction. Many visitors could be induced to move toward a region of the space that they normally avoided visiting. The authors also introduce a measure of human activity that combines different types of data to capture key aspects of human behavior in interactive spaces.

L. Marchesotti, S. Piva, and C. S. Regazzoni's "Structured Context Analysis Techniques in Biologically Inspired Ambient Intelligent Systems" explores techniques and related issues for the definition of a contextual knowledge in AmI systems. A logical structure for this kind of system, inspired by a neurobiological brain model, is proposed. In particular, the role and the importance of context awareness in the definition of an artificial organism showing adaptability, pervasiveness, and scalability features are described. Techniques for the definition of a multilayer context representation are explained and practically demonstrated with a test bed. The main innovative part of the system is a complex event-classification algorithm based on the self-organizing map. Event classification is obtained through the fusion of heterogeneous data coming from a set of sensors. Experimental results are presented on a simplified system prototype that has been set up in a computer science laboratory at the University of Genoa, Genoa, Italy. The laboratory has been equipped with two coupled charge device cameras with fields of view partially overlapped in such a way that they assure a complete coverage of the lab room, as well as a better observation through the use of the augmented data obtained in the overlapped area thanks to data duplication. In addition, a badge reader has been installed in the main entrance of the lab to collect the number of users log-ins within the lab zone, and mouse and keyboard activity sensors have been added to control user behavior. A hard-disk working sensor completes the set of sensors to watch the system inner state.

"Probabilistic Posture Classification for Human Behavior Analysis," presented by R. Cucchiara, C. Grana, A. Prati, and $\mathrm{R}$. Vezzani, is placed in the context of computer vision and ubiquitous multimedia access and it deals with the development of an automated system for human behavior analysis. The proposed system is able to classify the posture of a person and, consequently, detecting corresponding events and alarm situations, like a fall. The main innovative part of the system consists in the computation for each frame of the projection histograms of each person and on the related comparison with the probabilistic projection maps stored for each posture during the training phase. A tracking algorithm is applied to validate the obtained posture by tacking into account the reliability of the classification of the first phase. Moreover, the tracking algorithm is used to handle occlusions making the system particularly robust even in indoors environments. Extensive experimental results demonstrate a promising average accuracy of more than $95 \%$ in correctly classifying human postures, even in the case of challenging conditions.

"Dynamic Context Capture and Distributed Video Arrays for Intelligent Spaces," presented by M. M. Trivedi, K. S. Huang, and I. Mikic, describes an intelligent system able to extract and maintain an awareness of a wide range of events and human activities occurring in indoor intelligent spaces. The proposed system is based on visual data as they play an important role in developing accurate and useful representation of the static and dynamic states of an "intelligent" environment. Some of the key functionalities of the proposed system include, intruder detection, multiple person tracking, body pose and posture analysis, person identification, human-body modeling and movement analysis, and for integrated systems for intelligent meeting rooms, teleconferencing, or performance spaces. Particularly innovative parts of the paper include the use of omnidirectional video camera arrays, video-stream synchronization, multicamera-based multiperson tracking, event detection and event-based serving for "selective" attention, voxelization, and streaming-face recognition.

In "An Intelligent Fuzzy Agent Approach for Realizing Ambient Intelligence in Intelligent Inhabited Environments," a new learning approach for intelligent agents that are embedded in intelligent inhabited environments is presented by F. Doctor, H. Hagras, and V. Callaghan. Intelligent agents aim to provide ubiquitous computing intelligence in the environment supporting the activities of the user. The main innovative part of the paper is an unsupervised, data-driven, fuzzy technique that is used for extracting fuzzy membership functions and rules that represent particular user's behaviors in the environment. The user's learned behaviors can then be adapted online in a lifelong mode to satisfy the different user and system objectives. A real ubiquitous computing environment test bed represented by an intelligent dormitory has been used to perform real experiments in which the intelligent agent has learnt and adapted to the user's behavior during a period of five consecutive days. Experimental results compare the performances of the presented approach with other existing approaches and demonstrate the superiority of the proposed one while operating online in a life long mode.

N. Heo and P. K. Varshney's “Energy-Efficient Deployment of Intelligent Mobile Sensor Networks," discusses a set of distributed energy-efficient deployment algorithms for mobile sensors and intelligent devices that form an ambient intelligent network. The integration of several mobile sensors and/or intelligent devices which need to communicate with each other is an essential constraint of intelligence environments who would use smart infrastructures to improve the quality of life and safety in emergency situations. Organization and optimization of network resources are fundamental to provide ubiquitous communication for a longer duration in large-scale networks. The main innovative part of the paper consists in 
the presentation of three new deployment algorithms, which employ a synergistic combination of cluster structuring and a peer-to-peer deployment scheme. The first algorithm is proposed for mobile nodes when each node is equally important. The second one is proposed for wireless sensor network scenarios; it employs a clustering strategy to take advantage of local information and to reduce energy consumption. Finally, an energy-efficient deployment algorithm based on Voronoi diagrams is proposed. Performance of the presented algorithms is evaluated in terms of coverage, uniformity, and time and distance traveled till the algorithm converges.

In "Health-Status Monitoring Through Analysis of Behavioral Patterns," the work of T. Barger, D. Brown, and M. Alwan illustrates a good example of futuristic domotics. Their paper introduces the importance of developing solutions for the elderly to aid them in their own environment: the work discusses the first steps toward a virtual maid, capable of tracking the movements of people in a multiroom environment—such as a home-and a way of drawing statistics on the movements to identify critical situations where alerting might be necessary. Their method assumes the deployment of a number of motion sensors and the use of the new standard clustering techniques and Markov chains to build a record of the evolution in the scene.

In "Sketch-Based Image Matching Using Angular Partitioning," presented by A. Chalechale, G. Naghdy, and A. Mertins, a system for automatically comparing a hand-drawn rough black and white sketch with an existing database of full-color images (artworks and photographs). In particular, a new image similarity measure based on Fourier transform features which are rotation and scale invariant and which are robust against translation is introduced. This measure allows the system to work with images containing several complex objects in an inhomogeneous background and to allow detection of distortion in any given real-time image sequences. The system provides the user with options for either retrieving similar images in the database or ranking the quality of the sketch against a given standard, i.e., the original image model. Several experiments from different approaches demonstrate the validity of the proposed method in both the retrieval performance and the time required for feature extraction and search.

\section{Charting a Foreseeable Future}

Research and technology must go hand in hand to develop AmI systems. Technology must move forward to provide the backbone of the AmI system: fast seamless clusters of computers communicating at very high speed, sharing information acquired by highly sophisticated sensors and ambient-controlled real time. The current state of the art in information understanding and relaying will have to make huge steps forward to design algorithms capable of understanding and intuiting human actions and speech. Neither research nor technology are sufficient to implement AmI environments. Both are necessary to create the soul and body of a truly pervasive and distributed layer, capable of dealing with user requests, preempting the user and guiding the user in private and public enhanced spaces.

\author{
PaOlo Remagnino, Guest Editor \\ Digital Imaging Research Centre \\ Kingston University \\ Kingston Upon Thames, KT1 2EE, UK \\ p.remagnino@kingston.ac.uk \\ GIAN LUCA FORESTI, Guest Editor \\ Department of Mathematics and Computer Science \\ University of Udine \\ UDINE 33100, Italy \\ foresti@dimi.uniud.it
}

\section{REFERENCES}

[1] Proc. 1st Eur. Symp. Ambient Intell. (EUSAI'03), E. Aarts, R. Collier, E. can Loenen, and B. de Rutyer, Eds., Nov. 2003.

[2] P. Remagnino and G. L. Foresti, Proc. Workshop Ambient Intell., 8th Ital. Conf. Artif. Intell., Nov. 23-26, 2003.

[3] Proc. 2nd Eur. Symp. Ambient Intell. (EUSAI'04), P. Markopoulos, B. Eggen, E. Aarts, and J. L. Crowley, Eds., 2004.

[4] H. Hargras and V. Callaghan, Proc. IEE Int. Workshop Intell. Environ., Colchester, UK, 2005, to be published.

[5] Proc. 1st Int. Workshop Ubiquitous Smart Worlds, J. Ma, L. T. Yang, and D. Taniar, Eds., Taipei, Taiwan, Mar. 28-30, 2005, to be published.

[6] E. Aarts, "Ambient intelligence: A multimedia perspective," IEEE Trans. Multimedia, vol. 11, no. 1, Jan.-Mar. 12-19, 2004.

[7] G. Abowd, C. Atkeson, A. Bobick, I. Essa, B. MacIntyre, E. Mynatt, and T. Starner, "Living laboratories: The future computing environments group at the georgia institute of technology," in Proc. CHI, Eindhoven, The Netherlands, 2000, pp. 215-216.

[8] T. Basten, C. C. Geilen, and H. de Groot, Eds., Ambient Intelligence: Impact on Embedded Systems Design. Berlin, Germany: Springer-Verlag, 2003.

[9] M. H. Coen, "Design principles for intelligent environments," in Proc. 15th Nat./10th Conf. Artif. Intell./Innovative Applicat. Artif. Intell., Madison, WI, 1998, pp. 547-554.

[10] D. Wagelaar, A. Georges, P. Rigole, T. Clerckx, Y. Berbers, K. Coninx V. Jonckers, K. De Bosshere, D. Preuveneers, and J. Van den Bergh, "Toward an extensible context ontology for ambient intelligence," in Proc. 2nd Eur. Symp. Ambient Intell., Eindhoven, The Netherlands, Nov. 8-10, 2004, to be published.

[11] N. Daniele, M. Pezzin, S. Derivaz, J. Keignart, and P. rouzet, "Principle and motivations of UWB technology for high data rate WPAN applications," presented at the Smart Objects Conf., Grenoble, France, 2003.

[12] M. L. Dertouzos, "The future of computing," Sci. Amer., vol. 281, no. 2, pp. 52-55, 1999

[13] R. DeVaul, M. Sung, J. Gips, and A. Pentland, "Mithril 2003: Applications and architecture," in Proc. 7th Int. Symp. Wearable Comput., 2003, pp. $4-11$.

[14] F. Doctor, H. Hagras, and V. Callaghan, "A type-2 fuzzy embedded agent for ubiquitous computing environments," presented at the IEEE Int. Conf. Fuzzy Syst., 2004.

[15] I. Essa, "Computers seeing people," AI Mag., vol. 20, no. 2, pp. 69-82, 1999.

[16] S. K. Feiner, "Augmented reality: A new way of seeing," Sci. Amer, vol. 286, no. 4 , pp. $48-55,2002$

[17] J. Ferber, Multi-Agent Systems: An Introduction to Distributed Artificial Intelligence. Reading, MA: Addison-Wesley, 1999.

[18] G. L. Foresti and L. Snidaro, "A distributed sensor network for video surveillance of outdoors," in Multisensor Surveillance Systems: The Fusion Perspective, G. L. Foresti, C. S. Regazzoni, and P. Varshney, Eds. Norwell, MA: Kluwer, 2003, pp. 8-27.

[19] H. Hagras, V. Callaghan, M. Colley, G. Clarke, A. Pounds-Cornish, and H. Duman, "Creating an ambient-intelligence environment using embedded agents," IEEE Trans. Intell. Transp. Syst., vol. 19, no. 6, pp. 12-20, Nov.-Dec. 2004.

[20] I. E. Sutherland, "The ultimate display," in Proc. IFIPS Congress, vol. 2, 1965, pp. 506-508. 
[21] K. Ducatel, M. Bogdanowicz, F. Scapolo, J. Leijten, and J.-C. Burgelman, "Scenarios for Ambient Intelligence in 2010," IST Advisory Group Final Rep., Eur. Comm., Feb. 2001.

[22] K. Ducatel, M. Bogdanowicz, F. Scapolo, J. Leijten, and J.-C. Burgelman, "Ambient Intelligence: From Vision to Reality," IST Advisory Group Draft Rep., Eur. Comm., 2003.

[23] N. R. Jennings, "On agent-based software engineering," Artif. Intell., vol. 117, pp. 277-296, 2000.

[24] S. Kadamber and M. Iseli, "Fast online speaker/environment adaptationusing modified maximum likelihood stochastic matching," in Proc. Workshop Ambient Intell., 2003, pp. 131-141.

[25] C. D. Kidd, R. Orr, G. D. Abowd, C. G. Atkeson, I. A. Essa, B. MacIntyre, E. D. Mynatt, T. Starner, and W. Newstetter, "The aware home: A living laboratory for ubiquitous computing research," in Proc. 2nd Int. Workshop Coop. Buildings, 1999, pp. 191-198.

[26] SONY Interaction Laboratory. Interaction Lab. [Online]. Available: http://www.csl.sony.co.jp/IL/index.html

[27] L. Marcenaro, L. Marchesotti, F. Cella, and C. S. Regazzoni, "A distributed dynamic reconfigurable system for ambient intelligence," in Proc. Intell. Distributed Surveillance Syst., 2003, pp. 21-27.

[28] P. Marti and H. H. Lund, "Chapter scaling ambient intelligence: Compositional devices," in A Novel Paradigm. Berlin, Germany: SpringerVerlag, 2005, pp. 39-61.

[29] I. C. Millard, "Ambient Intelligence: The Contextually Aware Environment,", M.Phil. Transfer Rep., 2004.

[30] A. Nijholt, "Disappearing computers, social actors, and embodies agents," in Proc. Int. Conf. Cyberworld, 2003, pp. 128-134.

[31] _ "Meetings, gatherings, and events in smart environments," in Proc. ACM SIGGRAPH Int. Conf. Virtual-Reality Continuum Applicat. Industry, 2004, pp. 229-232.

[32] D. A. Norman, The Invisible Computer. Cambridge, MA: MIT Press, 1999.

[33] A. P. Pentland, "Smart rooms," Sci. Amer., vol. 274, no. 4, pp. 68-76, 1996.

[34] ——, "Wearable Intelligence," Scientif. Amer., vol. 276, no. 1, 1998.
[35] R. W. Picard, Affective Computing. Cambridge, MA: MIT Press, 1997.

[36] P. Remagnino, G. L. Foresti, and T. Ellis, Ambient Intelligence: A Novel Approach. Berlin, Germany: Springer-Verlag, 2005.

[37] B. Russell and K. Norvig, Artificial Intelligence: A Modern Approach. Englewood Cliffs, NJ: Prentice-Hall, 2003.

[38] N. Shadbolt, "Ambient intelligence," IEEE Trans. Intell. Transp. Syst., vol. 18, no. 4, pp. 2-3, Jul.-Aug. 2003.

[39] Y. Shoham, "Agent-oriented programming," Artif. Intell., vol. 60, pp. 51-92, 1993.

[40] M. Trivedi, K. Huang, and I. Mikic, "Intelligent environments and active camera networks," in Proc. IEEE Int. Conf. Syst., Man, Cybern., vol. 2, 2000, pp. 804-809.

[41] E. J. van Loenen, "On the role of graspable objects in the ambient intelligence paradigm," presented at the Smart Objects Conf., 2003.

[42] W. Verhaegh, E. Aarts, and J. Korst, Eds., Algorithms in Ambient Intelligence. Berlin, Germany: Springer-Verlag, 2003.

[43] W. Weber, "Ambient intelligence: Industrial research on a visionary concept," in Proc. Int. Symp. Low-Power Electron. Design, 2003, pp. 247-251.

[44] M. Weiser, "The computer for the twenty-first century," Sci. Amer, vol. 265, no. 3, pp. 94-104, 1991.

[45] - "Some computer science problems in ubiquitous computing," Commun. ACM, vol. 36, no. 7, pp. 74-84, 1993.

[46] M. Wooldridge, An Introduction to Multiagent Systems. Hoboken, NJ: Wiley, 2002.

[47] C. Wren, F. Sparacino, A. J. Azarbayejani, T. J. Darrell, J. W. Davis, T. E. Starner, A. Kotani, C. M. Chao, M. Hlavac, K. B. Russell, A. Bobick, and A. P. Pentland, "Perceptive spaces for performance and entertainment: Untethered interaction using computer vision and audition," Appl. Artif. Intell., vol. 11, no. 4, pp. 267-284, 1997.

[48] M. Xu, J. Orwell, and G. A. Jones, "Tracking football players with multiple cameras," in Proc. IEEE Int. Conf. Image Process., 2004, pp. 1935-1938.

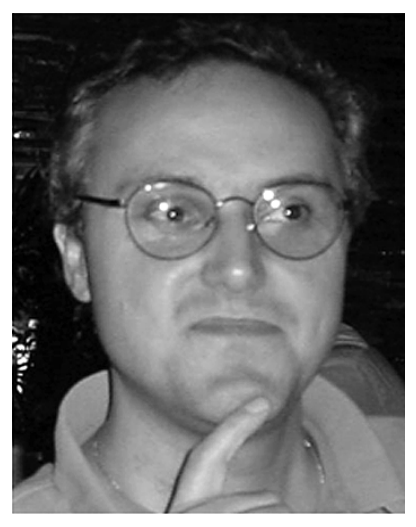

Paolo Remagnino (S'90-M'98) received the Laurea Diploma degree in electronic engineering from the University of Genoa, Genoa, Italy, in 1988 and the Ph.D. degree in computer vision from the University of Surrey, Surrey, UK, in 1993.

$\mathrm{He}$ is the author of more than 60 papers in the field of computer vision and artificial intelligence and he is the main editor of a book on visual surveillance techniques and a book on ambient intelligence (AmI). He is an active researcher with more than ten years experience in the field of computer vision and artificial intelligence. His current interests include computer vision, image processing, complex scene understanding, machine learning techniques, information fusion, and AmI.

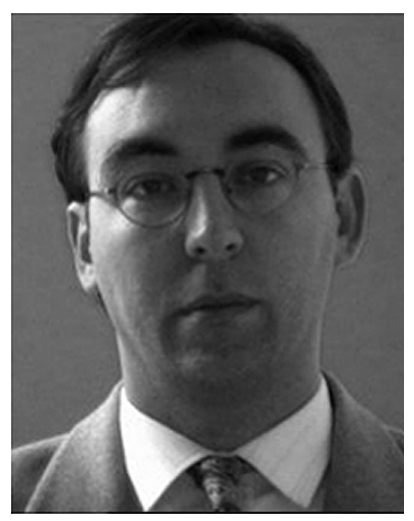

Gian Luca Foresti (S'93-M'95-SM'01) received the Laurea Diploma degree (cum laude) in electronic engineering and the Ph.D. degree in computer science from the University of Genoa, Genoa, Italy, in 1990 and in 1994, respectively.

Since 2000 he has been a Professor of computer science in the Department of Mathematics and Computer Science (DIMI), University of Udine, Udine, Italy, where he is also the Director of the AVIRES Lab. He is the author of more than 150 papers published in international journals and refereed international conferences. He has contributed to seven books in his area of interest and he is coauthor of Multimedia Systems for Visual-based Surveillance (Norwell, MA: Kluwer, 2000), Multisensor Surveillance Systems: The Fusion Perspective (Norwell, MA: Kluwer, 2003), and Ambient Intelligence: A Novel Paradigm (Berlin, Germany: Springer-Verlag, 2005). His main interests include computer vision and image processing, multisensor data fusion, artificial neural networks, and pattern recognition.

Prof. Foresti was recently the Guest Editor of a Special Issue of the Proceedings of IEEE on Video Communications, Processing and Understanding for Third Generation Surveillance Systems. He served as General Co-chair, Chairman, and Member of Technical and Program Committees at several conferences. Currently, he is the Finance Chair of the IEEE International Conference on Image Processing (ICIP) 2005. 